\title{
A karyotype study in two fish species belonging to Genus Neolissochilus found in Meghalaya, India
}

\author{
Raffealla Nongrum* \\ Department of Biotechnology, Assam Don Bosco University, Tapesia, Sonapur (Assam), \\ India \\ Rabindra Nath Bhuyan \\ Department of Fishery Science, St. Anthony's College, Shillong (Meghalaya), India \\ *Corresponding author. E mail: rafela88@gmail.com
}

\section{Article Info}

https://doi.org/10.31018/ jans.v13i3.2764

Received: June 17, 2021

Revised: September 8, 2021

Accepted: September 13, 2021

\section{How to Cite}

Nongrum, R. and Bhuyan, R. N. (2021). A karyotype study in two fish species belonging to Genus Neolissochilus found in Meghalaya, India. Journal of Applied and Natural Science, 13(3), 1127 - 1134. https://doi.org/10.31018/jans.v13i3.2764

\begin{abstract}
The karyomorphological study of two species of Mahseer belonging to the genus Neolissochilus, namely Neolissochilus hexagonolepis and $N$. hexastichus were carried out. The study revealed the basic chromosome number in both the Masheer species was observed to be 100 . However, the karyotype formula number varied among the species. N. hexagonolepis had a diploid chromosome number of 42 metacentric $(\mathrm{m}), 20$ submetacentric $(\mathrm{sm}), 8$ subtelocentric (st) and 30 telocentric (t) and $N$. hexastichus had a karyotypic formula of 32 metacentric $(\mathrm{m}), 22$ submetacentric $(\mathrm{sm}), 4$ subtelocentric (st) and 42 telocentric (t). This finding removed taxonomic confusion due to the differences in the chromosome number, the morphology of the chromosomes and chromosome formula between the two fish species of the genus and helped in distinctive and unblemished identification of the two species belonging to the genus Neolissochilus from Meghalaya, though they have a morphological similarity.
\end{abstract}

Keywords: Mahseer, Neolissochilus hexagonolepis, Neolissochilus hexastichus, Karyotype, Taxonomic

\section{INTRODUCTION}

Cyprinid fishes of the genus Neolissochilus are naturally found throughout tropical and subtropical areas of southern and south-eastern Asia (Rainboth, 1885). The importance of these mahseers is due to their delicacy, high protein content (Day, 1876) as food and as game fish. The fishes in angling tourism are gaining importance. Several organisations, through the development of sanctuaries, are working for their conservations (Joshi et al., 2018) in Meghalaya, in particular, such as establishing 54 sanctuaries till date since 2012 in Garo, Khasi and Jaintia Hills Districts (Dash et al., 2020). Neolissochilus hexagonolepis and $N$. hexastichus are cyprinids found in the rivers of Meghalaya, India. The Chocolate mahseer or $N$. hexagonolepis, one of the important fish species, is a highly esteemed food and game fish found in the North-Eastern Himalayan region, particularly in Meghalaya. The fish is considered a threatened species and needs special attention to conserve to increase its population in natural water bodies (International Union for Conservation of Nature, 2021). Three different water bodies, viz., River Khri of Umiam,
East Khasi Hills, River Umran and River Umraleng of Ri Bhoi District, Meghalaya, India were explored for the presence of mahseer species and studies on the water parameters of these rivers were found to be conducive for mahseer growth and propagation (Sarma and Bhuyan, 2007).

$N$. hexastichus, on the other hand, is found only in the Janiaw river of Mawsynram in East Khasi Hills District of Meghalaya during our survey and is a confusing species due to its similarities to Tor tor (Menon, 1974). Brown mahseer or $N$. hexastichus has been claimed as a valid species which has characteristics different from other species of Tor as the Tor characters in this species are very poorly represented (Day, 1878) and resurrected from synonymy Tor tor. $N$. hexastichus is survived with a small population in the river Diyung in Dima Hasao district in Assam, which is perhaps its last stronghold (Laskar, 2013; Kar and Khynriam, 2020). Hence, a thorough survey on the occurrence of this species in another locality of rivers of Meghalaya, India, is urgent.

A prominent study by Sen and Jayaram (1982) concluded that $N$. hexastichus can not be included under 
Tor genus and lips are thick with a continuous labial groove in Tor and an interrupted groove in Neolissochilus. Among the mahseer cyprinids, an easily distinguishable character is relatively large on their body (Desai, 2003) and Tor can be distinguished from other cyprinids by having a fleshy median mental lobe (Roberts, 1999). Due to the similarities in the morphometric characters and meristic counts, difficulties have been encountered in the correct identification of the mahseer belonging to Neolissochilus. This group of fishes and chromosome karyotype study and molecular techniques are required to resolve such ambiguities.

One of the important strategies for the conservation of fish is the artificial propagation of the species, either insitu or ex-situ, which helps run the rivers or other natural habitats. However, the first step for breeding programmes is the correct identification of a fish species with proper taxonomical tools. The most reliable taxonomic information should be collected from literature and extensive examination of specimens and taxonomic and systematics studies have a high standard from the rest of science (Vecchione et al., 2000). For evolutionary status, the main source of information is the morphology of the fish specimens. For evolutionary status, the main source of information is the morphology of the fish specimen.

The present study will provide a better insight into the presence of the particular fish species $N$. hexastichus in Meghalaya, India, and its differences from N. hexagonolepis, an abundant fish species found in many rivers of Meghalaya.

\section{MATERIALS AND METHODS}

The sampling of fishes ( $N$. hexagonolepis and N. hexasticus) was done from two places Janiaw River of East Khasi Hills District and Umraleng River of Jaintia Hills District of Meghalaya. A total of 6 fishes were used for karyotype since only a limited number of $N$. hexastichus species were obtained. The fishes were brought to the Fishery Science Department of St. Anthony's College and acclimatized in ponds and no Animal ethics approval is required for the capture of fishes since the healthy ones are kept for breeding programs.

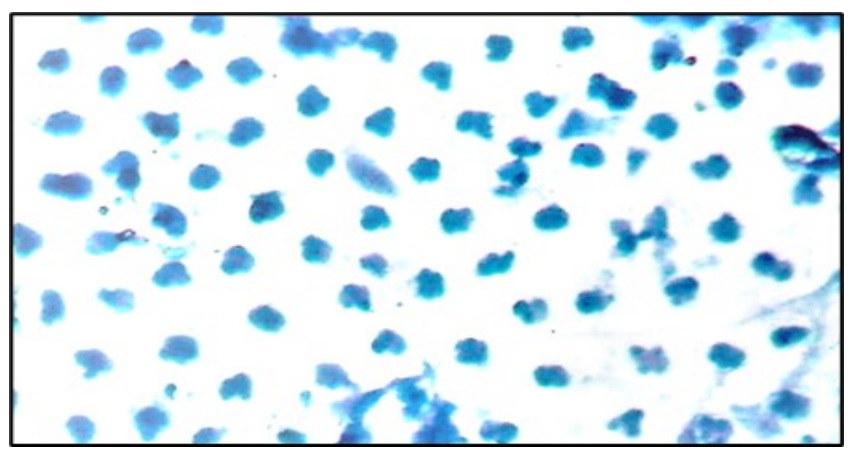

Morphometric characters and Meristic counts were employed to confirm the correct identification of fish species with the help of measuring length, weight, counting of fins and many specific characters (Cavalcanti et al., 1999) and the specific keys used are paper-based. The species were further supported by ZSI (Zoological Survey of India), Shillong, in which the samples from the Janiaw river and Umraleng river were sent for species confirmation.

The study of chromosomes through cytogenetic spreads was conducted in the two species of Neolissochilus. For Karyotypic studies, healthy fishes were considered and each fish was injected intramuscularly with 0.01 per cent Colchicine at a dose of $1 \mathrm{ml} / 100 \mathrm{~g}$ bodyweight of the fish. The fishes were kept in a wellaerated aquarium for 2 hours. The fishes were then anaesthetized using chloroform for 5 minutes. The gills and kidney tissues were isolated and immediately, the tissues were processed following the $\mathrm{KCl}$ Acetomethanol-flame drying method (Barat et al., 2012). The slides were then stained with 5 per cent Giemsa stain ( $\mathrm{pH} \mathrm{6.8)}$ for 2 hours, washed in distilled water, mounted in DPX (Dibutylphthalate Polystyrene Xylene). The slides were observed under Trinocular Microscope with immersion objective and images were taken using a Motic camera. The karyomorphological identification was made based on the length of the $p$ arm, length of the $q$ arm, arm ratio (length of the long arm to the short arm of the chromosome) calculated and separated as metacentric $(\mathrm{m})$, submetacentric $(\mathrm{sm})$, subtelocentric $(\mathrm{st})$ and telocentric (t) as per Levan et al. (1964).

\section{RESULTS AND DISCUSSION}

The cytogenetic spread of the light micrograph of both species is shown in Fig. 1. The chromosomes number was found to be 100 , which was consistent with the chromosome number in carps. During karyomorphological care was taken to measure only the visible and -defined chromosome arms. Table 1 and Table 2 represent the calculated $p$ arm, $q$ arm, arm ratio, centromeric index of $N$. hexagonolepis and $N$. hexastichus, respectively, based on which the chromosome type was then categorised

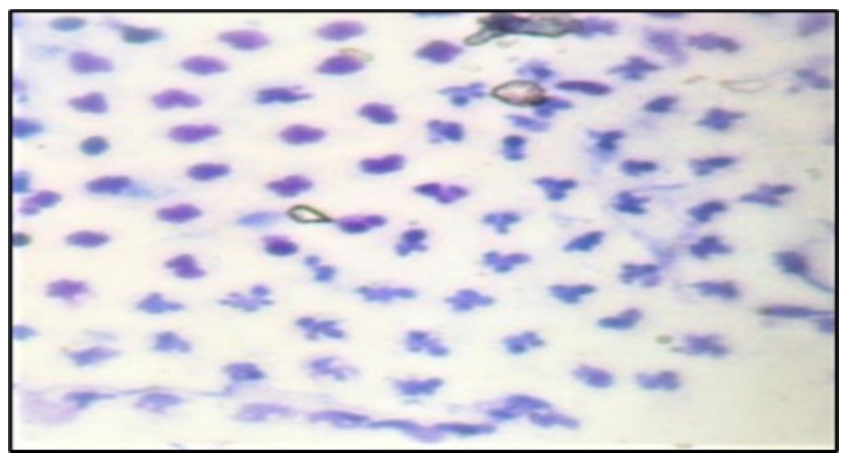

Fig.1. Light micrograph showing Cytogenetic spread 
This study is the first reported from the region and if available, the reports are scanty if at all available. It was found that the chromosome number in both the species was 100 and it was concluded that the number of chromosomes is conserved as seen in mahseer species of cyprinids. Since the chromosome size is small and usually abundant and has more contracted structures, studying and measuring fish chromosomes is somewhat more difficult than mammals (Suleyman et al., 2004; Saxena and Vasave, 2012). The most commonly occurring diploid number in family Cyprinidae is 50 , considered to be the modal number in the case of this family (Manna, 1983; 1984; Rishi, 1989) and also seen in the family Puntius (Ganai and Yousuf, 2011), which is valid over 80 per cent of metaphase spread. Sahoo et al. (2007) showed a karyotype of 32 metacentric $(\mathrm{m}), 16$ sub-metacentric $(\mathrm{sm}), 6$ sub-telocentric (st) and 46 telocentric $(\mathrm{t})$ on the karyotype analysis of $N$. hexagonolepis. However, the present study recorded 42 metacentric $(\mathrm{m}), 20$ submetacentric $(\mathrm{sm}), 8$ subtelocentric (st) and 30 telocentric (t) for $N$. hexagonolepis as shown in Table 1 and Figure 2, whereas for $N$. hexastichus it was 32 metacentric $(\mathrm{m}), 22$ submetacentric (sm), 4 subtelocentric (st), 42 telocentric (t) as shown in Table 2 and Figure 3. The presence of similar chromosome number $(2 n=100)$ with varied karyotypes in Mahseer species of Neolissochilus and Tor species suggests the evolution among both the species through

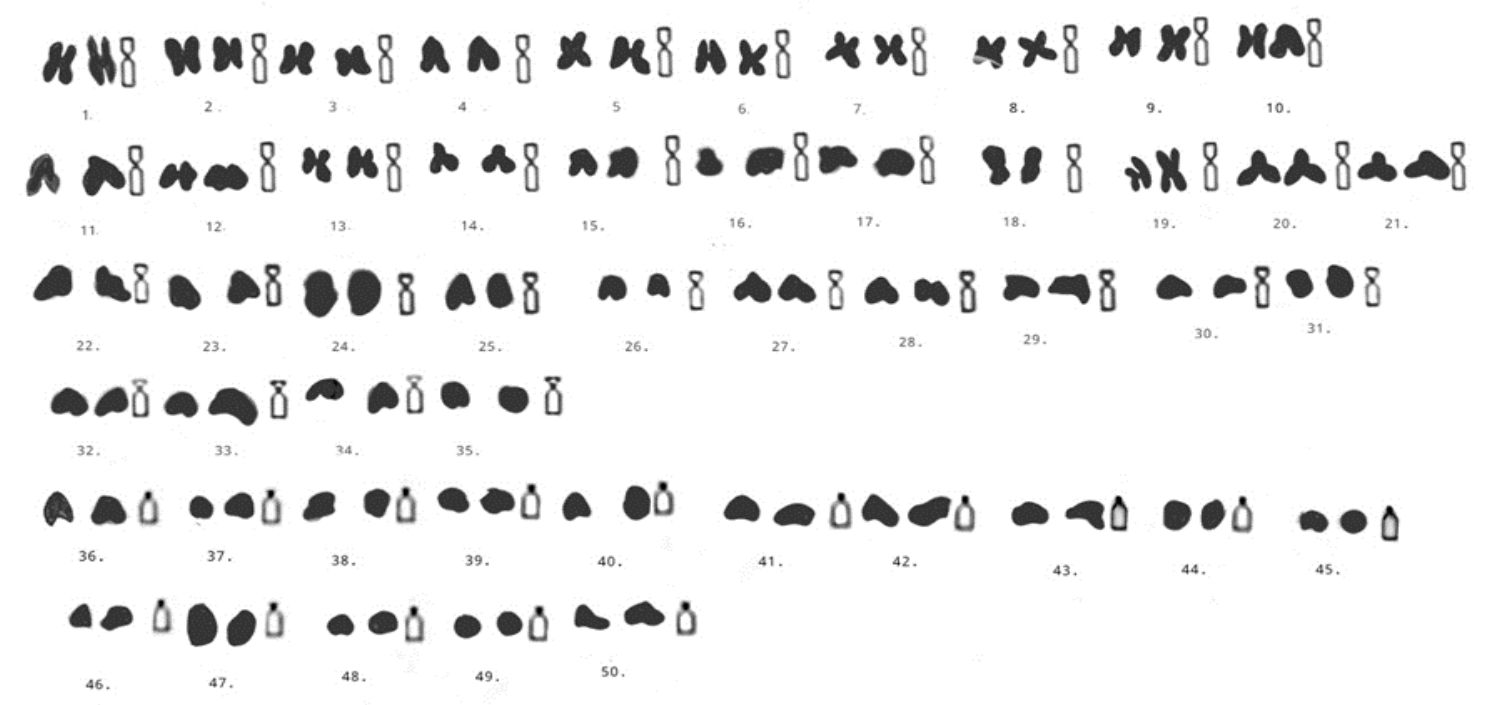

Fig. 2. Arrangements of chromosomes of $N$. hexagonolepis in pairs in according to their morphological appearances.

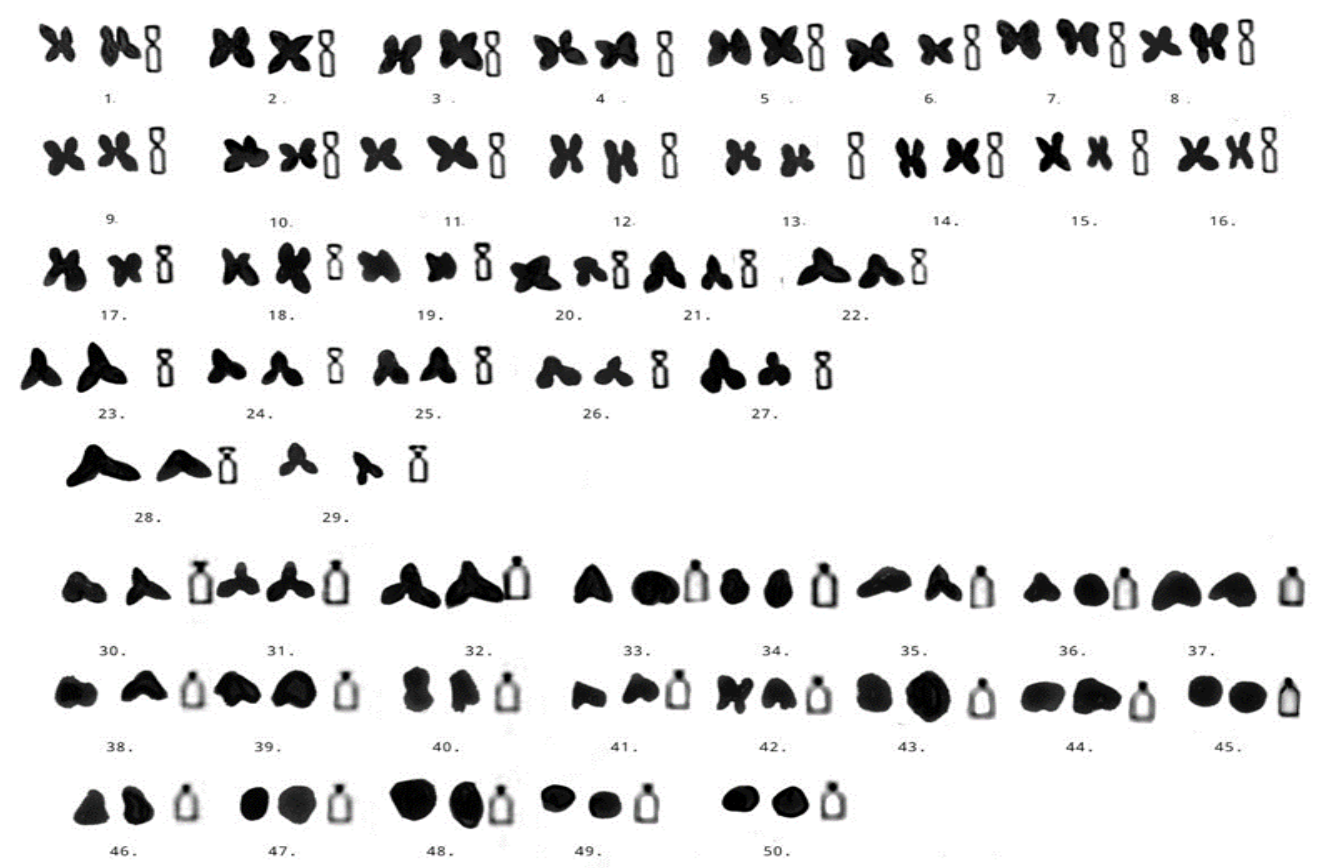

Fig. 3. Arrangements of chromosomes of $N$. hexastichus in pairs in according to their morphological appearances 
Nongrum, R. and Bhuyan, R. N. / J. Appl. \& Nat. Sci. 13(3), 1127 - 1134 (2021)

Table 1. Karyomorphology of $N$. hexagonolepis chromosomes.

\begin{tabular}{|c|c|c|c|c|c|c|}
\hline S.No & $p$ arm $(\mu m)$ & $q \operatorname{arm}(\mu \mathrm{m})$ & Arm ratio & $\begin{array}{l}\text { Total } \\
\text { length }(\mu \mathrm{m})\end{array}$ & $\begin{array}{l}\text { Centeromeric } \\
\text { index }\end{array}$ & $\begin{array}{l}\text { Chromosome } \\
\text { type }\end{array}$ \\
\hline 1 & 1.8 & 1.35 & 1.25 & 2.43 & 44.44444444 & $M$ \\
\hline 2 & 1.21 & 1.12 & 1.080357143 & 2.33 & 48.06866953 & $M$ \\
\hline 3 & 1.44 & 1.36 & 1.058823529 & 2.8 & 48.57142857 & $M$ \\
\hline 4 & 1.57 & 1.57 & 1 & 3.14 & 50 & $M$ \\
\hline 5 & 0.7 & 0.5 & 1.4 & 1.2 & 41.66666667 & $M$ \\
\hline 6 & 0.5 & 0.7 & 1.4 & 1.2 & 41.66666667 & $M$ \\
\hline 7 & 0.5 & 0.6 & 1.2 & 1.1 & 45.45454545 & $M$ \\
\hline 8 & 0.3 & 0.4 & 1.333333333 & 0.7 & 42.85714286 & $M$ \\
\hline 9 & 0.6 & 0.6 & 1 & 1.2 & 50 & $M$ \\
\hline 10 & 0.6 & 0.8 & 1.333333333 & 1.4 & 42.85714286 & $M$ \\
\hline 11 & 0.6 & 0.5 & 1.2 & 1.1 & 45.45454545 & M \\
\hline 12 & 0.4 & 0.8 & 2 & 1.2 & 33.33333333 & SM \\
\hline 13 & 1.1 & 1.0 & 1.1 & 2.1 & 47.61904762 & $\mathrm{M}$ \\
\hline 14 & 0.8 & 0.6 & 1.333333333 & 1.4 & 42.85714286 & $M$ \\
\hline 15 & 0.7 & 1.0 & 1.428571429 & 1.7 & 41.17647059 & M \\
\hline 16 & 0.4 & 0.7 & 1.75 & 1.1 & 36.36363636 & SM \\
\hline 17 & 0.7 & 0.7 & 1 & 1.4 & 50 & $\mathrm{M}$ \\
\hline 18 & 0.9 & 0.8 & 0.888888889 & 1.7 & 41.17647059 & M \\
\hline 19 & 0.5 & 1.0 & 2 & 1.5 & 60 & SM \\
\hline 20 & 0.6 & 0.4 & 1.5 & 1 & 40 & $\mathrm{M}$ \\
\hline 21 & 0.4 & 0.9 & 2.25 & 1.3 & 30.76923077 & SM \\
\hline 22 & 0.6 & 0.9 & 1.5 & 1.5 & 40 & $\mathrm{M}$ \\
\hline 23 & 0.4 & 0.5 & 1.25 & 0.9 & 44.44444444 & $M$ \\
\hline 24 & 0.6 & 0.6 & 1 & 1.2 & 50 & $M$ \\
\hline 25 & 0.7 & 0.9 & 1.285714286 & 1.6 & 43.75 & $M$ \\
\hline 26 & 0.6 & 0.7 & 1.166666667 & 1.3 & 46.15384615 & $M$ \\
\hline 27 & 0.6 & 0.6 & 1 & 1.2 & 50 & $M$ \\
\hline 28 & 0.6 & 0.4 & 1.5 & 1 & 40 & $M$ \\
\hline 29 & 0.7 & 0.5 & 1.4 & 1.2 & 41.66667 & $\mathrm{M}$ \\
\hline 30 & 0.4 & 0.7 & 1.75 & 1.1 & 36.36363636 & SM \\
\hline 31 & 0.2 & 0.8 & 2 & 1 & 20 & SM \\
\hline 32 & 0.5 & 0.6 & 1.333333333 & 1.1 & 45.45454545 & $\mathrm{M}$ \\
\hline 33 & 0.5 & 0.6 & 1.333333333 & 1.1 & 45.45454545 & $M$ \\
\hline 34 & 0.7 & 0.6 & 1.166666667 & 1.3 & 46.15384615 & $M$ \\
\hline 35 & 0.8 & 0.8 & 1 & 1.6 & 50 & $M$ \\
\hline 36 & 0.8 & 0.8 & 1 & 1.6 & 50 & $\mathrm{M}$ \\
\hline 37 & 0.7 & 0.2 & 3.5 & 0.9 & 22.22222222 & ST \\
\hline 38 & 0.8 & 0.9 & 1.125 & 1.7 & 47.05882353 & $\mathrm{M}$ \\
\hline 39 & 0.6 & 0.7 & 1.166666667 & 1.3 & 46.15384615 & $\mathrm{M}$ \\
\hline 40 & 0.5 & 0.4 & 1.25 & 0.9 & 44.44444444 & $\mathrm{M}$ \\
\hline 41 & 1.0 & 0.5 & 2 & 1.5 & 33.33333333 & SM \\
\hline 42 & 0.7 & 0.5 & 1.4 & 1.2 & 41.66666667 & $\mathrm{M}$ \\
\hline 43 & 1.4 & 0.5 & 2.8 & 1.9 & 26.31578947 & SM \\
\hline 44 & 0.6 & 0.7 & 1.166666667 & 1.3 & 46.15384615 & $\mathrm{M}$ \\
\hline 45 & 0.9 & 0.4 & 2.25 & 1.3 & 30.76923077 & SM \\
\hline 46 & 1.1 & 0.4 & 2.75 & 1.5 & 26.66666667 & SM \\
\hline 47 & 1.4 & 0.2 & 7 & 1.6 & 12.5 & ST \\
\hline 48 & 0.8 & 0.4 & 2 & 1.2 & 33.33333333 & SM \\
\hline 49 & 0.3 & 0.8 & 2.666666667 & 1.1 & 27.27272727 & SM \\
\hline 50 & 0.4 & 1.1 & 2.75 & 1.5 & 26.66666667 & SM \\
\hline 51 & 0.4 & 0.9 & 2.25 & 1.3 & 30.76923077 & SM \\
\hline 52 & 0.5 & 1.0 & 2 & 1.5 & 33.33333333 & SM \\
\hline
\end{tabular}


Nongrum, R. and Bhuyan, R. N. / J. Appl. \& Nat. Sci. 13(3), 1127 - 1134 (2021)

\begin{tabular}{|c|c|c|c|c|c|c|}
\hline 53 & 0.2 & 0.8 & 4 & 1 & 20 & ST \\
\hline 54 & 1.2 & 0.4 & 3 & 1.6 & 25 & ST \\
\hline 55 & 0.2 & 0.7 & 3.5 & 0.9 & 22.22222222 & ST \\
\hline 56 & 0.4 & 1.2 & 3 & 1.6 & 25 & ST \\
\hline 57 & 0.4 & 0.5 & 1.25 & 0.9 & 44.44444444 & M \\
\hline 58 & 0.3 & 1.2 & 4 & 1.5 & 20 & ST \\
\hline 59 & 1.0 & 1.0 & 1 & 2 & 50 & $M$ \\
\hline 60 & 1.0 & 1.0 & 1 & 2 & 50 & M \\
\hline 61 & & 0.7 & 0.7 & & & $\mathrm{~T}$ \\
\hline 62 & & 1.1 & 1.1 & & & $T$ \\
\hline 63 & & 0.5 & 0.5 & & & $\mathrm{~T}$ \\
\hline 64 & & 0.9 & 0.9 & & & $\mathrm{~T}$ \\
\hline 65 & & 1.1 & 1.1 & & & $T$ \\
\hline 66 & & 0.7 & 0.7 & & & $T$ \\
\hline 67 & & 1.0 & 1 & & & $T$ \\
\hline 68 & & 0.9 & 0.9 & & & T \\
\hline 69 & & 1.2 & 1.2 & & & $\mathrm{~T}$ \\
\hline 70 & & 0.6 & 0.6 & & & T \\
\hline 71 & & 0.4 & 0.4 & & & T \\
\hline 72 & & 0.5 & 0.5 & & & T \\
\hline 73 & & 0.3 & 0.3 & & & $T$ \\
\hline 74 & & 0.5 & 0.5 & & & $T$ \\
\hline 75 & & 0.9 & 0.9 & & & $\mathrm{~T}$ \\
\hline 76 & 1.2 & 0.6 & 2 & 1.8 & 33.33333333 & SM \\
\hline 77 & 1.0 & 0.4 & 2.5 & 1.4 & 28.57142857 & SM \\
\hline 78 & & 0.7 & 0.7 & & & T \\
\hline 79 & & 1.1 & 1.1 & & & T \\
\hline 80 & & 0.7 & 1.285714286 & 1.6 & 43.75 & $M$ \\
\hline 81 & 0.9 & 0.4 & 2 & 1.2 & 33.33333333 & SM \\
\hline 82 & & 0.8 & 0.8 & & & $T$ \\
\hline 83 & & 0.8 & 0.8 & & & $T$ \\
\hline 84 & & 0.4 & 0.4 & & & $\mathrm{~T}$ \\
\hline 85 & & 0.6 & 0.6 & & & $\mathrm{~T}$ \\
\hline 86 & 0.8 & 0.4 & 2 & 1.2 & 33.33333333 & SM \\
\hline 87 & & 0.4 & 0.4 & & & $T$ \\
\hline 88 & & 0.9 & 0.9 & & & $\mathrm{~T}$ \\
\hline 89 & & 0.7 & 0.7 & & & $T$ \\
\hline 90 & & 0.9 & 0.9 & & & $T$ \\
\hline 91 & & 0.8 & 0.8 & & & $\mathrm{~T}$ \\
\hline 92 & 0.3 & 0.9 & 3 & 1.2 & 25 & ST \\
\hline 93 & 1.1 & 1.1 & 1 & 2.2 & 50 & M \\
\hline 94 & 0.5 & 0.7 & 1.4 & 1.2 & 41.66666667 & $M$ \\
\hline 95 & 0.6 & 0.8 & 1.333333333 & 1.4 & 42.85714286 & M \\
\hline 96 & & 0.6 & 0.6 & & & $\mathrm{~T}$ \\
\hline 97 & & 0.7 & 0.7 & & & $T$ \\
\hline 98 & & 1.0 & 1 & & & $T$ \\
\hline 99 & & 0.9 & 0.9 & & & T \\
\hline 100 & 0.4 & 0.8 & 2 & 1.2 & 33.33333333 & SM \\
\hline
\end{tabular}


Nongrum, R. and Bhuyan, R. N. / J. Appl. \& Nat. Sci. 13(3), 1127 - 1134 (2021)

Table 2. Karyomorphology of $N$. hexastichus chromosomes.

\begin{tabular}{|c|c|c|c|c|c|c|}
\hline S.No & $\begin{array}{l}\text { p arm } \\
(\mu \mathrm{m})\end{array}$ & $\begin{array}{l}\text { q arm } \\
(\mu \mathrm{m})\end{array}$ & Arm ratio & $\begin{array}{l}\text { Total length } \\
(\mu \mathrm{m})\end{array}$ & $\begin{array}{l}\text { Centeromeric } \\
\text { index }\end{array}$ & $\begin{array}{l}\text { Chromosome } \\
\text { type }\end{array}$ \\
\hline 1 & 0.4 & 0.4 & 1 & 0.8 & 50 & $M$ \\
\hline 2 & 0.7 & 0.3 & 2.333333333 & 1 & 30 & SM \\
\hline 3 & 1.1 & 0.6 & 1.833333333 & 1.7 & 35.29411765 & SM \\
\hline 4 & 0.8 & 0.7 & 1.142857143 & 1.5 & 46.66666667 & $\mathrm{M}$ \\
\hline 5 & 0.5 & 0.7 & 1.142857143 & 1.2 & 41.66666667 & $M$ \\
\hline 6 & 1.0 & 0.6 & 1.666666667 & 1.6 & 37.5 & $M$ \\
\hline 7 & 0.5 & 0.6 & 1.2 & 1.1 & 54.54545455 & $\mathrm{M}$ \\
\hline 8 & 0.6 & 0.3 & 2 & 0.9 & 33.33333333 & SM \\
\hline 9 & 0.5 & 0.4 & 1.25 & 0.9 & 44.44444444 & $\mathrm{M}$ \\
\hline 10 & 0.8 & 0.6 & 1.333333333 & 1.4 & 42.85714286 & $M$ \\
\hline 11 & 0.7 & 0.6 & 1.166666667 & 1.3 & 46.15384615 & $M$ \\
\hline 12 & 0.5 & 0.4 & 1.25 & 0.9 & 44.44444444 & $M$ \\
\hline 13 & 0.8 & 0.6 & 1.333333333 & 1.4 & 42.85714286 & $M$ \\
\hline 14 & 0.9 & 0.3 & 3 & 1.2 & 25 & ST \\
\hline 15 & 0.6 & 0.6 & 1 & 1.2 & 50 & $\mathrm{M}$ \\
\hline 16 & 1.0 & 0.4 & 2.5 & 1.4 & 28.57142857 & SM \\
\hline 17 & 0.4 & 0.9 & 2.25 & 1.3 & 30.76923077 & SM \\
\hline 18 & 0.6 & 0.7 & 1.166666667 & 1.3 & 46.15384615 & $\mathrm{M}$ \\
\hline 19 & 0.9 & 0.4 & 2.25 & 1.3 & 30.76923077 & SM \\
\hline 20 & 0.6 & 0.4 & 1.5 & 1 & 40 & $\mathrm{M}$ \\
\hline 21 & 0.2 & 1.3 & 6.5 & 1.5 & 13.33333333 & ST \\
\hline 22 & 0.3 & 1.2 & 4 & 1.5 & 20 & ST \\
\hline 23 & 0.4 & 0.9 & 2.25 & 1.3 & 30.76923077 & SM \\
\hline 24 & 0.5 & 0.5 & 1 & 1 & 50 & $\mathrm{M}$ \\
\hline 25 & 0.4 & 0.7 & 1.75 & 1.1 & 36.36363636 & SM \\
\hline 26 & 0.7 & 0.1 & 7 & 0.8 & 12.5 & ST \\
\hline 27 & 0.4 & 1.2 & 3 & 1.6 & 25 & SM \\
\hline 28 & & 1.0 & 1 & & & $\mathrm{~T}$ \\
\hline 29 & & 0.4 & 0.4 & & & $\mathrm{~T}$ \\
\hline 30 & & 0.6 & 0.6 & & & $\mathrm{~T}$ \\
\hline 31 & & 1.3 & 1.3 & & & $\mathrm{~T}$ \\
\hline 32 & & 0.7 & 0.7 & & & $\mathrm{~T}$ \\
\hline 33 & & 0.9 & 0.9 & & & $\mathrm{~T}$ \\
\hline 34 & & 1.0 & 1 & & & $\mathrm{~T}$ \\
\hline 35 & & 1.1 & 1.1 & & & $\mathrm{~T}$ \\
\hline 36 & & 0.9 & 0.9 & & & $\mathrm{~T}$ \\
\hline 37 & & 0.8 & 0.8 & & & $\mathrm{~T}$ \\
\hline 38 & 0.7 & 0.6 & 1.166666667 & 1.3 & 46.15384615 & $\mathrm{M}$ \\
\hline 39 & 0.6 & 0.8 & 2 & 1.2 & 50 & SM \\
\hline 40 & 0.5 & 0.9 & 1.5 & 1.5 & 33.33333333 & $\mathrm{M}$ \\
\hline 41 & & 0.8 & 0.8 & & & $\mathrm{~T}$ \\
\hline 42 & & 1.0 & 1 & & & $\mathrm{~T}$ \\
\hline 43 & & 0.7 & 0.7 & & & $\mathrm{~T}$ \\
\hline 44 & & 0.9 & 0.9 & & & $\mathrm{~T}$ \\
\hline 45 & & 1.3 & 1.3 & & & $\mathrm{~T}$ \\
\hline 46 & & 1.4 & 1.4 & & & $\mathrm{~T}$ \\
\hline 47 & & 0.9 & 0.9 & & & $\mathrm{~T}$ \\
\hline 48 & & 1.1 & 1.1 & & & $\mathrm{~T}$ \\
\hline 49 & & 1.8 & 1.8 & & & $\mathrm{~T}$ \\
\hline 50 & & 1.0 & 1 & & & $\mathrm{~T}$ \\
\hline 51 & & 0.6 & 0.6 & & & $\mathrm{~T}$ \\
\hline 52 & & 0.8 & 0.8 & & & $\mathrm{~T}$ \\
\hline
\end{tabular}


Nongrum, R. and Bhuyan, R. N. / J. Appl. \& Nat. Sci. 13(3), 1127 - 1134 (2021)

\begin{tabular}{|c|c|c|c|c|c|c|}
\hline 53 & & 1.1 & 1.1 & & & $\mathrm{~T}$ \\
\hline 54 & & 0.9 & 0.9 & & & $\mathrm{~T}$ \\
\hline 55 & & 1.3 & 1.3 & & & $\mathrm{~T}$ \\
\hline 56 & & 1.0 & 1 & & & $\mathrm{~T}$ \\
\hline 57 & & 1.1 & 1.1 & & & $\mathrm{~T}$ \\
\hline 58 & & 1.5 & 1.5 & & & $\mathrm{~T}$ \\
\hline 59 & & 1.1 & 1.1 & & & $\mathrm{~T}$ \\
\hline 60 & & 0.7 & 0.7 & & & $\mathrm{~T}$ \\
\hline 61 & 0.4 & 0.7 & 1.75 & 1.1 & 36.36363636 & SM \\
\hline 62 & 0.4 & 0.8 & 2 & 1.2 & 33.33333333 & SM \\
\hline 63 & 0.6 & 0.7 & 1.166666667 & 1.3 & 46.15384615 & $\mathrm{M}$ \\
\hline 64 & 0.6 & 0.4 & 1.5 & 1 & 40 & $M$ \\
\hline 65 & 0.6 & 0.4 & 1.5 & 1 & 40 & $M$ \\
\hline 66 & 0.6 & 0.4 & 1.5 & 1 & 60 & $M$ \\
\hline 67 & 0.9 & 0.6 & 1.5 & 1.5 & 60 & $M$ \\
\hline 68 & 1.2 & 0.6 & 2 & 1.8 & 33.33333333 & SM \\
\hline 69 & 0.8 & 0.6 & 1.333333333 & 1.4 & 42.85714286 & $\mathrm{M}$ \\
\hline 70 & 0.6 & 0.5 & 1.2 & 1.1 & 45.45454545 & $M$ \\
\hline 71 & 0.9 & 0.7 & 1.285714286 & 1.6 & 43.75 & M \\
\hline 72 & 0.5 & 1.1 & 2.2 & 1.6 & 31.25 & SM \\
\hline 73 & 0.5 & 0.6 & 1.2 & 1.1 & 45.45454545 & $\mathrm{M}$ \\
\hline 74 & 1.1 & 0.6 & 1.833333333 & 1.7 & 35.29411765 & SM \\
\hline 75 & 0.5 & 0.6 & 1.2 & 1.1 & 45.45454545 & $\mathrm{M}$ \\
\hline 76 & 0.8 & 0.3 & 2.666666667 & 1.1 & 27.27272727 & SM \\
\hline 77 & 0.8 & 0.3 & 2.666666667 & 1.1 & 27.27272727 & SM \\
\hline 78 & 0.3 & 0.6 & 2 & 0.9 & 33.33333333 & SM \\
\hline 79 & 0.5 & 0.3 & 1.666666667 & 0.8 & 37.5 & $\mathrm{M}$ \\
\hline 80 & 0.4 & 0.6 & 1.5 & 1 & 60 & M \\
\hline 81 & & 1.2 & 1.2 & & & $\mathrm{~T}$ \\
\hline 82 & & 0.9 & 0.9 & & & $\mathrm{~T}$ \\
\hline 83 & & 0.6 & 0.6 & & & $\mathrm{~T}$ \\
\hline 84 & & 1.4 & 1.4 & & & $\mathrm{~T}$ \\
\hline 85 & 0.7 & 0.5 & 1.4 & 1.2 & 41.66666667 & $\mathrm{M}$ \\
\hline 86 & 0.4 & 0.9 & 2.25 & 1.3 & 69.23076923 & SM \\
\hline 87 & 0.7 & 0.5 & 1.4 & 1.2 & 41.66666667 & $\mathrm{M}$ \\
\hline 88 & & 1.2 & 1.2 & & & $\mathrm{~T}$ \\
\hline 89 & & 0.7 & 0.7 & & & $\mathrm{~T}$ \\
\hline 90 & & 0.4 & 0.4 & & & $\mathrm{~T}$ \\
\hline 91 & & 0.9 & 0.9 & & & $\mathrm{~T}$ \\
\hline 92 & 1.3 & 0.6 & 2.166666667 & 1.9 & 31.57894737 & SM \\
\hline 93 & & 1.3 & 1.3 & & & $\mathrm{~T}$ \\
\hline 94 & & 1.0 & 1 & & & $\mathrm{~T}$ \\
\hline 95 & 0.4 & 0.5 & 1.25 & 0.9 & 44.44444444 & SM \\
\hline 96 & 0.9 & 0.8 & 2 & 1.7 & 47.05882353 & SM \\
\hline 97 & 0.9 & 0.8 & 1.142857143 & 1.5 & 47.05882 & $\mathrm{M}$ \\
\hline 98 & & 0.7 & 0.7 & & & $\mathrm{~T}$ \\
\hline 99 & & 0.7 & 0.7 & & & $\mathrm{~T}$ \\
\hline 100 & 0.7 & 0.6 & 1.166666667 & 1.3 & 51.42857143 & $M$ \\
\hline
\end{tabular}


pericentric inversions and/or heterochromatic processes (Mani et al., 2013). and the evolution and systematics are considered robust tools in resolving taxonomic uncertainties (Mani et al., 2010).

\section{Conclusion}

They are distinctly two different species $N$. hexagonolepis and $N$. hexastichus based on the number of morphologically different chromosomes, which has a support of meristic characteristics. The study will provide insight into using a cytogenetic study to identify the fish species population of mahseer rich flowing rivers of Meghalaya, India. In addition, further analysis of studying chromosome evolution through sophisticated tools such as FISH or Fluorescence in Situ Hybridization karyotype and distribution of constitutive heterochromatin, the findings which can be used in the phylogenetic study of the different species of Neolissochilus.

\section{Conflict of interest}

The authors declare that they have no conflict of interest.

\section{REFERENCES}

1. Cavalcanti, M. J., Montelso, L. R. and Lopes, P. R. D (1999). Landmark based Morphometric analysis in selected species of serranid fishes (Perciformes: Teleostei). Zoological Studies, 38 (3), 287-84.

2. Barat, A., Ali, S., Khandelwal, S. and Sahoo, P. K. (2012). Genetic characterization of two cold water fishes from Kumaun Hills, Uttarkhand. Caryologia, 65, 311-315.

3. Dash, P., Tandel, R. S., Baruah, D. and Sarma, D. (2020) Mahseer sanctuaries of Meghalaya: A conservation and recreational perspective. Aquaculture Asia Magazine, 24 (1).

4. Day, F. (1876). On some of the fishes of the Deccan. Zoological Journal of the Linean Society, 12, 565-578.

5. Day, F. (1878). The Fishes of India, London, 556-582.

6. Desai, V. R. (2003). Synopsis of biological data on the Tor mahseer, Tor tor (Hamilton, 1822). FAO Fisheries Synopsis, 158.

7. Ganai, F. A. and Yousuf, A. R. (2011). A karyological analysis of Puntius conchonius (Hamilton, 1822) (Pisces, Cyprinidae), a new cytotype from Dal Lake Srinagar Kashmir, J and K, India. Int. J. Fish. Aquat. 3, 213-217.

8. International Union for Conservation of Nature (2021). International Union for Conservation of Nature Retrieved from https://www.iucnredlist.org, accessed February 2021.

9. Joshi, K. D., Das, S. C. S., Pathak, R. K., Khan, A., Sarkar, U. K and Roy, K. (2018). Pattern of reproductive biology of the endangered golden mahseer Tor putitora (Hamilton, 1822) with special reference to regional climate change implications on breeding phenology from lesser Himalayan region, India. Journal of Applied Animal Research, 46 (1),1289-1295.

10. Kar, D. and Khynriam, D. (2020). On a recent pioneering taxonomic study of the fishes from rivers Diyung, Vomvadung, Khualzangvadung, Tuikoi and Mahur in Dima Hasao district of Assam (India). Transylv. Rev. Syst. Ecol. Res., 22.3.

11. Laskar, B. A. (2013). Threat Identification and Population Assessment of Neolissochilus Hexastichus- An Endemic Mahseer Fish in North-East India Vis-À-Vis Conservation Actions., 2nd RSG Final Technical Report.

12. Levan, A., Fredga, K. and Sandberg, A. A. (1964). Nomenclature of centeromeric positions on chromosomes. Heriditas, 52, p. 201-220.

13. Mani, I., Kumar, R., Kushwaha, B., Singh, M., Nagpure, N. S., Srivastava, P. K. and Lakra, W. S. (2010). Cytogenetic characterization of an endemic mahseer, Tor mohal mohanadicus (David, 1953; Teleostei: Cyprinidae). Nucleus, 53, 109-114.

14. Mani, I., Kumar, R., Singh, M., Kushwaha, B., Nagpure, N. S., Srivastava, P. K. and Lakra, W. S. (2013). Chromosomal distribution of constitutive heterochromatin in eight species of mahseers (Family: Cyprinidae) from India. Indian J. Biotechnol., 12, 178-186.

15. Manna, G. K. (1983). Cytogenetic studies on fishes and amphibia. In: Genetical Research in India. XVth Int. Cong. Genet. Publication and Information Division, ICAR, New Delhi, 242-273.

16. Manna, G. K. (1984). Progress in fish cytogenetics. Nucleus, 27, 203-231.

17. Menon, A. G. K. (1974). A check-list of Fishes of the Himalayan and the Indo-Gangetic Plains. J. Indl. Fish. Soc. India, spl. Pub, 1, 45-47.

18. Rainboth, W. J. (1885). Neolissochilus, a new genus of South Asian cyprinid fishes. Beaufortia, 35, 25-35.

19. Rishi, K. K. (1989) Current status of fish cytogenetics. In: Fish Genetics in India. (eds. Das, P and Jhingran, V.G). Today and Tomorrows Printers and Publishers, New Delhi, 1-20.

20. Roberts, T. R. (1999). Fishes of the cyprinid genus Tor in the Nam Theun watershed (Mekong basin) of Laos, with decription of a new species. The Raffles Bulletin of Zoology, 47, p. 225-236.

21. Sahoo, P. K., Nanda, P. and Barat, A. (2007). Karyotypic Analysis of Neolissocheilus hexagonolepis (McClelland), Puntius ticto (Ham.) and P. chola (Ham.) (Family: Cyprinidae, Pisces). Cytologia, 72, 409-413.

22. Sarma, D. and Bhuyan, R. N. (2007). Chocolate Mahseer (Neolissochilus hexagonolepis) Icon of Meghalaya Rivers. Fishing Chimes, 26, 116-117.

23. Saxena, A. and Vasave, S. (2012). Diversity of Fish chromosomes. Aquafind, Aqua fish database est. 1991.

24. Sen, T.K. and Jayaram, K.C. (1982). The Mahseer fish of India-a review. Rec. Zoological Survey of India. Misc. Publ. Occassional paper, 39, 38.

25. Suleyman, G., Ahmet, C., Ilhan, S. and Bertal, K. (2004). Karyotype analysis in Alburnus heckeli (Battalgil, 1943) from Lake Hazer. Turk. J. Vet. Anim. Sci., 28, 309-314.

26. Vecchione, M., Mickevich, M. F., Fauchald, K., Collette, B. B., Williams, A. B., Munroe, T. A. and Young, R. E. (2000). Importance of assessing taxonomic adequacy in determining fishing effects on marine biodiversity. ICES J. Mar. Sci., 57, 677-681. 